\title{
EARLY DEVELOPMENTAL OUTCOME FOLLOWING CONVULSIVE STATUS EPILEPTICUS
}

Investigators at the Developmental Cognitive Neurosciences Unit, Institute of Child Health, London, other centers in the UK, and Dartmouth School of Medicine, NH, USA, prospectively recruited children aged between 1 and 42 months from North London who had at least one episode of convulsive status epilepticus (CSE) and classified them as prolonged febrile seizures (PFS) or nonfebrile CSE. Of 54 patients receiving neuropsychological and MR imaging tests within 6 weeks (mean 38 days) of CSE (baseline) and at 1-year follow-up, 27 had PFS (mean age 18.4 months) and 27 had nonfebrile CSE (mean age 15.5 months). Children with nonfebrile CSE had similar seizure characteristics but a worse developmental outcome than children with PFS $(p<0.002)$. The PFS group had a worse developmental outcome than healthy controls (mean age 20.49 months) ( $\mathrm{p}=0.002)$. Tests at 1-year follow-up showed no significant improvement from baseline. Seizure characteristics were not predictive of performance. (Martinos MM, Yoong M, Patil S, et al. Early developmental outcomes in children following convulsive status epilepticus: A longitudinal study. Epilepsia 2013 Jun;54(6):1012-9). (Resp.: Dr Marina M Martinos. E-mail: m.martinos@ich.ucl.ac.uk).

COMMENT. Following convulsive status epilepticus (CSE), children including those with prolonged febrile seizures (PFS) are developmentally delayed. The impairments in development are still present 1 year post CSE, suggesting that premorbid abilities may be overshadowing any transient direct effects of CSE itself on outcome.

FS duration and Developmental Delay. The FEBSTAT Study Team investigated the association between FS duration and baseline characteristics of development in 158 children with a first FS, median duration 4 minutes (Hesdorffer DC, et al. Ann Neurol 2011 Jul;70(1):93-100). One population was identified that accounted for $82.3 \%$ of FSs and had a mean duration of $3.8 \mathrm{~min}$ (short FS) and a second population accounting for $17.7 \%$ of FSs with a mean duration of 39.8 min (long FS). Long FSs were significantly associated with developmental delay $(\mathrm{p}=0.01)$ and younger age at first FS $(p=0.048)$. The data provide support for redefining a simple febrile seizure, limiting the duration to no longer than $10 \mathrm{~min}$.

\section{SLEEP DISORDERS AND EPILEPSY}

An investigator from University of Oxford, UK, reviews the effect of sleep disorders on epilepsy and the effects of epilepsy on sleep. The occurrence of seizures is modified by the circadian sleep-wake cycle, the stage of sleep, sleep deprivation, and obstructive sleep apnea. Certain seizures, such as Rolandic epilepsy, are accentuated during sleep. Nocturnal epilepsy may cause arousals in sleep, and circadian sleep-wake rhythms may be disrupted and cause impaired cognitive function and behavior during the day. Antiepileptic drugs may have indirect effects on sleep. For example, barbiturates cause daytime sleepiness, and phenytoin causes insomnia.

Sleep disorders confused with epilepsy include parasomnias, arousal disorders, sleepwalking, sleep terrors, rhythmic movement disorders (headbanging), nightmares, 